\title{
Global checklist of species of Grania (Clitellata: Enchytraeidae) with remarks on their geographic distribution
}

\author{
Alessandro PRANTONI ${ }^{1, *}$, Paulo C. LANA ${ }^{2} \&$ Christer ERSÉUS ${ }^{3}$ \\ ${ }^{1,2}$ Center for Marine Studies, Federal University of Paraná, Av. Beira Mar, s/n, \\ 83255-976 Pontal do Paraná, Paraná, Brazil. \\ ${ }^{3}$ Department of Biological and Environmental Sciences, University of Gothenburg, \\ Box 463, SE-405 30 Göteborg, Sweden. \\ *Corresponding author: aprantoni@gmail.com \\ 2Email:1ana@ufpr.br \\ 33Email: christer.erseus@bioenv.gu.se \\ ${ }^{1}$ urn:1sid:zoobank.org:author:3FE88982-3151-43AF-BC1C-5C8F863729B7 \\ ${ }^{2}$ urn:lsid:zoobank.org:author:B5A74B14-A681-4D2A-8248-67DE8FCE9F7B \\ ${ }^{3}$ urn:lsid:zoobank.org:author:D98F606A-B273-4F50-95F5-C35F17B12C85
}

\begin{abstract}
A checklist of all currently accepted species of Grania Southern, 1913 (Annelida, Clitellata, Enchytraeidae) is presented. The genus is widespread over the world and comprises 81 species described to date. Remarks on their geographical distribution, habitat, synonymies and museum catalogue numbers are provided.
\end{abstract}

Keywords. Species list, Annelida, marine clitellates, geographic distribution, interstitial fauna.

Prantoni A., Lana P.C. \& Erséus C. 2017. Global checklist of species of Grania (Clitellata: Enchytraeidae) with remarks on their geographic distribution. European Journal of Taxonomy 391: 1-44. https://doi.org/10.5852/ ejt.2017.391

\section{Introduction}

Grania Southern, 1913 is a morphologically homogeneous and easily recognizable genus of marine Enchytraeidae Vejdovský, 1879 with a worldwide distribution. The worms are typically small, only a few mm long, with a filiform, nematode-like body and a characteristic pattern of few, stout chaetae (Fig.1). Most species live interstitially in intertidal or subtidal sands, but a few taxa are known from the deep sea.

The genus was originally established for specimens of Grania maricola Southern, 1913, sampled from the west coast of Ireland. Later on, Pierantoni (1915) regarded Grania maricola and his own species, Enchytraeus macrochaetus Pierantoni, 1901, at the time referred to Michaelsena Ude, 1896 (Pierantoni 1903), as synonyms. Stephenson (1930) instead considered them as separate species within Michaelsena. Nielsen \& Christensen (1959) pointed out, however, that Stephenson's concept of Michaelsena was artificial, and they transferred both species to the genus Enchytraeus Henle, 1837. 
Two authors independently re-established the genus Grania in 1966. Kennedy (1966) re-described G. maricola and G. macrochaeta and described G. americana Kennedy, 1966 from off the coast of Florida, while Lasserre (1966) transferred Michaelsena postclitellochaeta Knöllner, 1935 to Grania. One year later, considering the similarities among G. maricola, G. macrochaeta and G. americana, Lassserre (1967) proposed to divide G. macrochaeta into four subspecies, G. m. macrochaeta, G. m. americana, G. m. maricola and G. m. roscoffensis Lasserre, 1967. This situation lasted for about a decade, after which many additional species of Grania were described from different parts of the world (Erséus \& Lasserre 1976; Erséus 1977, 1980, 1990; Jamieson 1977; Coates \& Erséus 1985; Coates 1990; Rota \& Erséus 1996, 1997; Locke \& Coates 1998, 1999; De Wit \& Erséus 2007; Rota et al. 2007; De Wit et al. 2009; Prantoni et al. 2016), bringing a better understanding of species-specific characters and raising the subspecies proposed by Lasserre (1967) back to species status (Coates 1984; De Wit 2010). More recently, Prantoni et al. (2016) updated the phylogeny of the genus together with the descriptions and genetic data of nine new species, for the first time including species from Africa and the east coast of South America (Brazil) (see Discussion).

The genus is morphologically and genetically well separated from other enchytraeid genera (Erséus et al. 2010; De Wit et al. 2011; Prantoni et al. 2016).

To provide a global taxonomic overview of Grania, the present checklist summarizes historical and recently published data, including all valid species described to date. Moreover, the general geographical distribution patterns of the various species are briefly discussed.

\section{Material and methods}

The checklist is arranged in chronological order and based on a bibliographic survey. All records of Grania from published papers and monographs were reviewed. When available, additional information on habitat, geographical distribution and museum catalogue numbers is included.

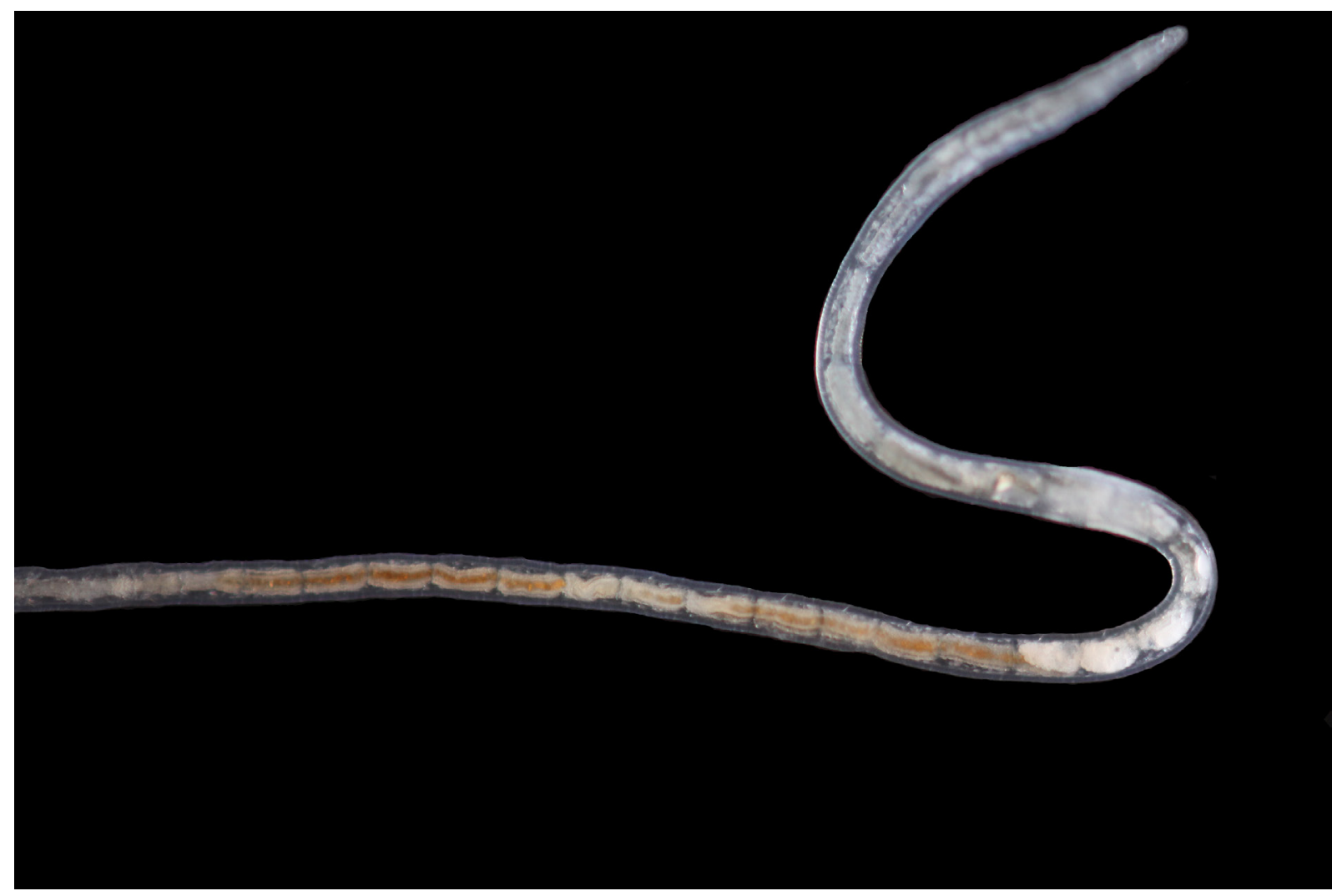

Fig. 1. Specimen of Grania chilensis Prantoni, De Wit \& Erséus, 2016. Photograph by Pierre De Wit. 


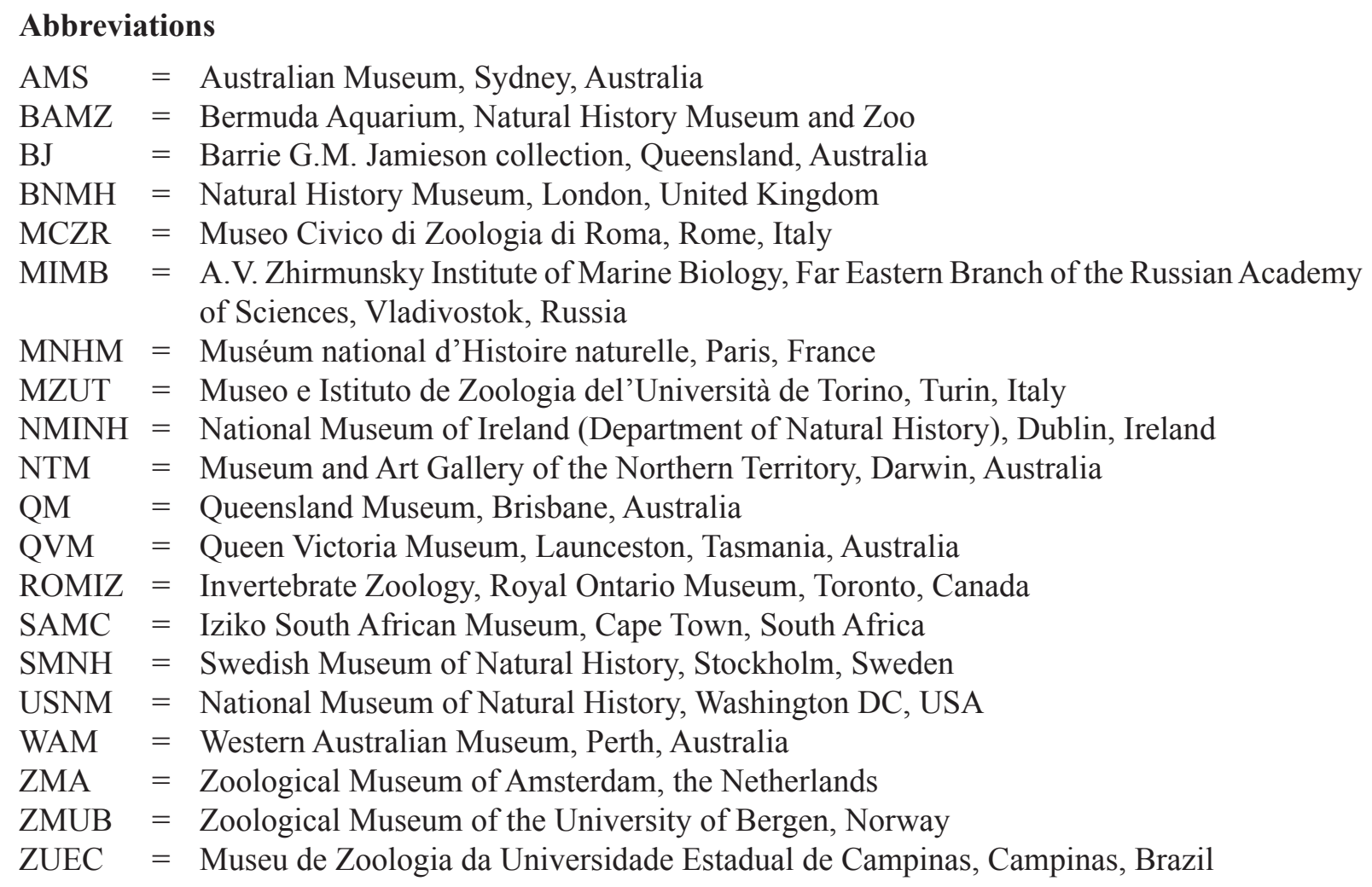

\section{Results}

We report 81 currently accepted species of Grania, of which 49 are known from the Southern and 32 from the Northern Hemisphere. The only species found in both hemispheres (Atlantic Ocean) is the deep-sea Grania atlantica Coates \& Erséus, 1985. Almost half of the known southern species (24) occur in Australia. On the other hand, 15 of the 32 species described from the Northern Hemisphere to date are European (Fig. 1).

\section{List of all described species of Grania in the World}

Phylum Annelida Lamarck, 1809

Class Clitellata Michaelsen, 1919

Order Enchytraeida Vejdovský, 1879

Family Enchytraeidae Vejdovský, 1879

Genus Grania Southern, 1913

1. Grania monochaeta (Michaelsen, 1888)

Enchytraeus monochaetus Michaelsen, 1888: 66-68, figs 6a-c.

Enchytraeus monochaetus - Beddard 1895: 339. — Michaelsen 1900: 91.

Marionina monochaeta - Nielsen \& Christensen 1959: 109.

Grania monochaeta - Lasserre 1967: 279-280. — Rota \& Erséus 1997: 29-34, fig. 2, tab. 1 (lectotype designation).

Hemigrania monochaeta - Lasserre 1971: 454. 
non Michaelsena monochaeta - Michaelsen 1921: 3. - Stephenson 1932: 263, fig. 14 (see Rota \& Erséus 1997).

non Grania monochaeta - Erséus \& Lasserre 1977: 299-300, figs 1a-d.

\section{Type material}

Lectotype

UNITED KINGDOM: South Georgia (MZUT 123.1).

\section{Paralectotypes}

UNITED KINGDOM: South Georgia (MZUT Olig. Coll. 123.2-123.4 ).

\section{Other material}

UNITED KINGDOM: South Georgia (SMNH 362).

\section{Type locality}

UNITED KINGDOM: South Georgia, SW Atlantic Ocean.

\section{Habitat}

Intertidal, subtidal to $20 \mathrm{~m}$, shelly detritus, among the roots of seaweeds and in the canal system of sponges.

\section{Distribution}

Only known from the type locality.

2. Grania macrochaeta (Pierantoni, 1901)

Enchytraeus macrochaetus Pierantoni, 1901: 201-202.

Michaelsena macrochaeta - Pierantoni 1903: 409-444, figs 1-28; 1915: 48-50. - Stephenson 1930: 776-777.

Enchytraeus macrochaetus - Nielsen \& Christensen 1959: 89-91, tab. 9.

Grania macrochaeta - Kennedy 1966: 403-404 (redescription). - Lasserre 1966: 312-314 (redescription). — Erséus 1974: 90-93, tab. 1. - Rota 1995: tab. 2.

Grania macrochaeta macrochaeta - Lasserre 1967: 280. — Erséus \& Lasserre 1976: 122, fig. 1, tab. 1.

\section{Type material}

Not designated.

\section{Other material}

ITALY: Bay of Naples (MNHM AH 61-63). This refers to three specimens collected in June, 1967 by J. Renaud-Mornant and examined by Erséus \& Lasserre (1976).

\section{Type locality}

ITALY: Bay of Naples.

\section{Habitat}

Subtidal, 4-13 m, coarse sand.

\section{Distribution}

Only known from the type locality. 


\section{Grania paucispina (Eisen, 1904)}

Michaelsena paucispina Eisen, 1904: 74, fig. 43.

Michaelsena paucispina - Michaelsen 1907: 130.

Marionina paucispina - Nielsen \& Christensen 1959: 109. — Lasserre 1971: 454.

Grania paucispina - Erséus \& Lasserre 1976: 127. — Coates \& Erséus 1980: 1037-1038, fig. 1. —

Coates \& Ellis 1981: 2134.

\section{Type material}

Typus perditus - specimen dissolutus. According to Coates \& Erséus (1980), the type material deposited in the California Academy of Science in San Francisco was destroyed during the earthquake and fire in 1906.

\section{Other material}

UNITED STATES: California (USNM 58906-58907).

CANADA: British Columbia (USNM 58907).

\section{Type locality}

UNITED STATES: Santa Barbara, California.

\section{Habitat}

In muddy sand, 2-6 m, with much organic material, brackish water.

\section{Distribution}

California, USA and British Columbia, Canada.

4. Grania principissae (Michaelsen, 1907)

Michaelsena principissae Michaelsen, 1907: 129-131, pl. 1, figs 1-2.

Hemigrania principissae - Lasserre 1971: 454.

Grania principissae - Erséus \& Lasserre 1976: 128. — Coates 1990: 28-30, figs 2, 8.

\section{Type material}

Typus amissus.

\section{Other material}

AUSTRALIA: Western Australia, Princess Royal Harbour (WAM 69-89, ROMIZ 11277).

\section{Type locality}

AUSTRALIA: Princess Royal Harbour, Albany area, Western Australia.

\section{Habitat}

Intertidal, sand with organic debris accumulated in mussel bed.

\section{Distribution}

Only known from the type locality. 
5. Grania maricola Southern, 1913

Grania maricola Southern, 1913: 14, figs 1-7.

Michaelsena macrochaeta - Pierantoni 1915 (part): 48-50.

Michaelsena maricola - Stephenson 1930: 776-777.

Enchytraeus maricolus - Nielsen \& Christensen 1959: 89-91, tab. 9.

Grania maricola - Kennedy 1966: 400-402, fig. 2 (redescription). — Lasserre 1966: 312-314 (redescription). - Erséus 1974: 90-93; 1976: 35, tab. 3. - Erséus \& Lasserre 1976: 124-125, figs 4-5, tab. 1. - Bonomi \& Erséus 1984: 209, tab. 1. - Coates 1984: 40, fig. 7a, tab. 1. Rodriguez 1986: 82-83, fig. 2. - Rota 1995: tab. 2. — Locke \& Coates 1998: 1111-1112, tab. 1. — De Wit 2006: 25. — van Haaren 2016: 140-141, figs 20, 56.

Grania macrochaeta maricola - Lasserre 1967: 280.

\section{Type material}

\section{Holotype}

IRELAND: County Kerry (NMINH 1913.415).

Paratypes

IRELAND: County Kerry (NMINH 1909.151, NMINH 1914.313).

\section{Type locality}

IRELAND: County Kerry.

\section{Habitat}

Subtidal, 8-80 m, coarse shell sand and shell gravel.

\section{Distribution}

Ireland, Iberian Peninsula, Italy, the Netherlands, Norway and Sweden.

6. Grania postclitellochaeta (Knöllner, 1935)

Michaelsena postclitellochaeta Knöllner, 1935: 449-455, figs 19-25, tab. 1.

Michaelsena postclitellochaeta - Hagen 1954: 12-13.

Marionina postclitellochaeta - Nielsen \& Christensen 1959: 109-110, tab. 11.

Grania postclitellochaeta postclitellochaeta - Lasserre 1966: 299-300, 312-314, tabs 1-2. - Erséus 1976: 35, tab. 3. - Erséus \& Lasserre 1976: 124, tab. 1.

Hemigrania postclitellochaeta - Lasserre 1971: 454-456, fig. 3c.

Grania postclitellochaeta - Kossmagk-Stephan 1983: 598. - Rota \& Erséus 2003: 232-234, figs 10c-d. — van Haaren 2016: 141-143, figs 21, 23, 25-26, 57.

\section{Type material}

Not designated.

\section{Other material}

FRANCE: Bassin d'Arcachon (SMNH 45646-45651, MCZR Oligochaeta 0116-0117); Gulf of Biscay (MNHM AH 66-68; see Erséus \& Lasserre 1976).

NORTH SEA: SMNH 45652-45654, MCZR Oligochaeta 0118.

ICELAND: Hafnafjörðdur (SMNH 45655-45660, MCZR Oligochaeta 0119; see Rota \& Erséus 2003). 
NORWAY: Tromsø (SMNH 45661-45665, MCZR Oligochaeta 0120; see Rota \& Erséus 2003); Bergen (SMNH 107746-107749; see De Wit \& Erséus 2010).

SWEDEN: Koster Islands (SMNH 107730, 107736, 107738-107745, 108220; see De Wit \& Erséus 2010).

\section{Type locality}

GERMANY: Kiel Bay.

\section{Habitat}

Intertidal, subtidal, 20-100 $\mathrm{m}$ in coarse shell sand, shell gravel, sometimes in brackish water (Baltic Sea), tolerating a wide range of salinity (11-35 ppt).

\section{Distribution}

Baltic Sea, Iceland (see Rota \& Erséus 2003), North Sea (Germany, the Netherlands and Belgium), France, Norway and Sweden.

\section{Remarks}

According to van Haaren (2016), it is not possible to distinguish Grania postclitellochaeta from G. occulta De Wit \& Erséus, 2010 and G. ovitheca Erséus, 1977 on morphological grounds only.

\section{Grania americana Kennedy, 1966}

Grania americana Kennedy, 1966: 404-405, fig. 3.

Grania macrochaeta americana - Lasserre 1967: 78-280.

Grania americana - Erséus 1974: 90-93, tab. 1. - Healy \& Coates 1999: 111, 114, tab. 1. — Locke \& Coates 1999: 598-623, figs 16-20 (redescription); 2000: 619-620, 625-626, figs 4a, 5. —Locke 2000: 83-93, figs 1-14.

Grania americana - Erséus \& Lasserre 1976: p. 123 (nomen dubium, see Erséus \& Lasserre 1976; Locke \& Coates 1999).

\section{Type material}

\section{Holotype}

BAHAMAS: North Bimini (USNM 33005).

Paratype

BAHAMAS: North Bimini (USNM 33039).

\section{Other material}

BAHAMAS: Pearl Island (BMAZ 1999180 009).

BERMUDA: Ferry Reach (USNM 185957).

UNITED STATES: Hutchinson Island (USNM 185958).

BELIZE: Carrie Bow Cay (USNM 185959-185960).

\section{Type locality}

BAHAMAS: North Bimini. 


\section{Habitat}

Intertidal, subtidal to $10 \mathrm{~m}$, fine to coarse sand.

\section{Distribution}

Belize, Bermuda, Bahamas, Florida.

8. Grania roscoffensis Lasserre, 1967

Grania macrochaeta roscoffensis Lasserre, 1967: 277-280.

Grania roscoffensis - Erséus 1974: 90-93, tab. 1. - Erséus \& Lasserre 1976: 125, fig. 6, tab. 1 (amended). — Coates 1984: 49. — Rota \& Erséus 2003: 218-221, figs 3a-c, 4.

non Grania roscoffensis — Erséus 1977: 294, tab. 1 (see Rota \& Erséus 2003).

\section{Type material}

\section{Holotype}

FRANCE: Roscoff, harbour (MNHM AH 64).

\section{Paratype}

The originally designated paratype AH 65 belongs to another species (see Rota \& Erséus 2003).

\section{Other material}

FRANCE: Roscoff, harbour (SMNH 45614).

SPAIN: Canary Islands (SMNH 45615, ZMAV.OL 9345).

\section{Type locality}

FRANCE: Roscoff, harbour.

\section{Habitat}

Intertidal, coarse sand and gravel.

\section{Distribution}

France, Sweden and Canary Islands.

\section{Grania pusilla Erséus, 1974}

Grania pusilla Erséus, 1974: 87-94, figs 1-6, tab. 1.

Grania pusilla - Erséus 1976: 34, tab. 3. - De Wit et al. 2011: 513, figs 1-5, tab. 1.

Grania macrochaeta pusilla - Erséus \& Lasserre 1976: 122, fig. 2. — Erséus 1977: 294, tab. 1. — Coates 1984: 49. — Kossmagk-Stephan 1985: 77-78.

non Grania pusilla - Locke \& Coates 1998: 1107-1112, figs 6-12 (see Rota \& Erséus 2003).

\section{Type material}

\section{Holotype}

NORWAY: Vågegrunnen (ZMUB 55050).

\section{Paratype}

NORWAY: Vågegrunnen (ZMUB 55051). 


\section{Other material}

SWEDEN: Koster Islands (SMNH 107775-107788).

NORWAY: Bergen (SMNH 107789-107796).

\section{Type locality}

NORWAY: Vågegrunnen, Hjeltefjorden.

\section{Habitat}

Subtidal, 35-500 m, fine to coarse shelly sand.

\section{Distribution}

West coasts of Norway and Sweden, Morocco.

10. Grania bermudensis Erséus \& Lasserre, 1976

Grania macrochaeta bermudensis Erséus \& Lasserre, 1976: 122-124, fig. 3 tab. 1.

Grania macrochaeta bermudensis - Erséus \& Lasserre 1976: 453. — Coates 1984: 49, fig. 8a.

Grania bermudensis - Locke \& Coates 1999: 609-614, figs 6, 12-15, tab. 1; 2000: 619-621, 626, fig. $6 \mathrm{c}$.

\section{Type material}

\section{Holotype}

BERMUDA: Castle Island (USNM 53202).

\section{Paratype}

BERMUDA: Castle Island (USNM 53203).

\section{Type locality}

BERMUDA: Castle Island.

\section{Habitat}

Subtidal, 8-15 m, medium to coarse coral sand and gravel.

\section{Distribution}

Only known from Bermuda.

\section{Grania longiducta Erséus \& Lasserre, 1976}

Grania postclitellochaeta longiducta Erséus \& Lasserre, 1976: 127, fig. 7, tab. 1.

Hemigrania postclitellochaeta - Lasserre 1971: 454-456, fig 3a-b, d .

Grania postclitellochaeta longiducta - Erséus 1977: 296-297. — Coates 1984: 49.

Grania longiducta - Coates \& Erséus 1985: 113-114, fig. 8. - Diaz et al. 1987: tab. 1, 3. — Locke \& Coates 2000: 619, 625.

\section{Type material}

\section{Holotype}

UNITED STATES: Massachusetts, Cape Cod Bay (USNM 43482).

\section{Paratype}

UNITED STATES: Massachusetts, Cape Cod Bay (USNM 53201). 
Type locality

UNITED STATES: Cape Cod Bay, Massachusetts.

\section{Habitat}

Subtidal, $42-78 \mathrm{~m}$, medium to coarse sand.

\section{Distribution}

Cape Cod Bay, Massachusetts, Georges Bank (SE of Massachusetts), off the coast of New Jersey, Delaware and Maryland, USA.

\section{Grania monospermatheca Erséus \& Lasserre, 1976}

Grania monospermatheca Erséus \& Lasserre, 1976: 127, fig. 9, tab. 1.

Grania monospermatheca - Coates 1984: 49, fig. 8b. — Coates \& Erséus 1985: 114-115, fig. 9. — Diaz et al. 1987: tab. 1-3. - Locke \& Coates 2000: 619, 626-628.

\section{Type material}

\section{Holotype}

UNITED STATES: Massachusetts, Cape Cod Bay (USNM 53204).

\section{Paratype}

UNITED STATES: Massachusetts, Cape Cod Bay (USNM 53205).

\section{Type locality}

UNITED STATES: Cape Cod Bay, Massachusetts.

\section{Habitat}

Subtidal, 3-48 m, fine to coarse well-sorted sands and sand mixed with shell or shell gravel.

\section{Distribution}

Along North American Atlantic coast, from Cape Cod Bay, Massachusetts to Biscayne Bay, Florida, USA.

\section{Grania variochaeta Erséus \& Lasserre, 1976}

Grania variochaeta Erséus \& Lasserre, 1976: 125-126, figs 10-11, tab. 1.

Grania variochaeta - Erséus 1976: 35, tab. 3; 1977: 297-298, tab. 1. — Coates 1984: 46, fig. 6. — Rota \& Erséus 2003: 211, 234-235, fig. 11. — van Haaren 2016: 143-144, fig. 58.

\section{Type material}

\section{Holotype}

SWEDEN: Kosterfjorden (SMNH 3132).

\section{Paratypes}

SWEDEN: Kosterfjorden (SMNH 3133-3136).

\section{Type locality}

SWEDEN: Kosterfjorden, Bohuslän. 


\section{Habitat}

Subtidal, 20-140 m, heterogeneous sand.

\section{Distribution}

West coast of Norway and Sweden, the Netherlands.

\section{Grania ovitheca Erséus, 1977}

Grania ovitheca Erséus, 1977: 125, figs 5-7.

Grania ovitheca - Bonomi \& Erséus 1984: 209, tab. 1. - Rota 1995: tab. 2. — Rota \& Erséus 2003: 230-233, figs 10a-b. — De Wit \& Erséus 2010: 286-291.

\section{Type material}

\section{Holotype}

SWEDEN: Kosterfjorden (SMNH 3071).

\section{Paratypes}

SWEDEN: Kosterfjorden (SMNH 3072-3073).

\section{Other material}

SWEDEN: off Gullmar Fjord (SMNH 107753, 107755-107758, 107760-107768).

\section{Type locality}

SWEDEN: Kosterfjorden, Bohuslän.

\section{Habitat}

Subtidal, 15-30 m, shell sand with gravel and pebbles or coarse sand with stones, pebbles and shells.

\section{Distribution}

West coasts of Norway and Sweden, Italy.

\section{Grania trichaeta Jamieson, 1977}

Grania macrochaeta trichaeta Jamieson, 1977: 345-347, fig. 5, pl. 1 g.

Grania macrochaeta trichaeta - Coates 1984: 46, fig. 5a.

Grania trichaeta - De Wit et al. 2009: 28-30, figs 8-9, 10a-e (redescription).

\section{Type material}

\section{Holotype}

AUSTRALIA: Queensland, Wistari Reef (QM 8863).

\section{Paratypes}

AUSTRALIA: Queensland, Wistari Reef (QM 8866, BNMH 1976.1.21-23, BJ 1975.7.76-78); Queensland, Heron Reef (BJ 1975.7.74-75, BJ 1975.7.84).

\section{Other material}

AUSTRALIA: Queensland, Lizard Island (AMS W.35554-W.35559, SMNH 105540-105559); Queensland, Heron Island (SMNH 105560-105584). 
Type locality

AUSTRALIA: Wistari Reef, Great Barrier Reef, Queensland.

\section{Habitat}

Intertidal, subtidal to $7 \mathrm{~m}$, fine to medium heterogeneous sand.

\section{Distribution}

Lizard Island, Heron Island and Wistari Reef, Great Barrier Reef, Queensland, Australia.

\section{Grania pacifica Shurova, 1979}

Grania pacifica Shurova, 1979: 84-86, fig. 6.

\section{Type material}

\section{Holotype}

RUSSIA: Iturup Island, Sea of Okhotsk (MIMB 16017).

\section{Type locality}

RUSSIA: Sea of Okhotsk, Iturup Island.

\section{Habitat}

Subtidal, 15-20 m, gravelly sediment.

\section{Distribution}

Only known from the type locality.

\section{Grania incerta Coates \& Erséus, 1980}

Grania incerta Coates \& Erséus, 1980: 1038-1040, fig. 2.

Grania incerta - Coates \& Ellis 1981: 2134-2135. — Coates 1984: 46, fig. 4.

\section{Type material}

\section{Holotype}

UNITED STATES: California, Santa Barbara (USNM 58908).

\section{Paratypes}

UNITED STATES: California, Santa Barbara (USNM 58909).

CANADA: British Columbia, Rennison Island (USNM 58910).

\section{Type locality}

UNITED STATES: Santa Barbara, California.

\section{Habitat}

Subtidal, 3-17 m, well-sorted fine sand.

\section{Distribution}

California, USA and British Columbia, Canada. 
18. Grania parvitheca Erséus, 1980

Grania parvitheca Erséus, 1980: 27-28, fig. 1.

Type material

Holotype

UNITED KINGDOM: Ascension Island (USNM 58738).

\section{Paratype}

UNITED KINGDOM: Ascension Island (USNM 58739).

\section{Type locality}

UNITED KINGDOM: Ascension Island, S Atlantic Ocean.

\section{Habitat}

Intertidal, among rocks and clumps of tubes of Sabellariidae.

\section{Distribution}

Only known from the type locality.

19. Grania atlantica Coates \& Erséus, 1985

Grania atlantica Coates \& Erséus, 1985: 112-113, fig. 7.

Grania atlantica - Diaz et al. 1987: 222-224, tabs 1, 3-4. - Locke \& Coates 2000: 619, 626. — Rota \& Erséus 1996: 182; 2003: 210-211, 235-237, fig. 10e. — Erséus \& Rota 2003: 898, tab. 1.

\section{Type material}

\section{Holotype}

UNITED STATES: off Massachusetts (USNM 96503).

\section{Paratypes}

UNITED STATES: off Massachusetts (USNM 96504-96508).

\section{Type locality}

UNITED STATES: off Massachusetts.

\section{Habitat}

Continental slope, 744-1796 m, fine ooze to silty deep-sea sediments.

\section{Distribution}

Widely distributed in the Atlantic Ocean from $48^{\circ} 35.4^{\prime} \mathrm{N}$ to $09^{\circ} 05^{\prime} \mathrm{S}$ in the east and from $39^{\circ} 51.2^{\prime} \mathrm{N}$ to $70^{\circ} 40.8^{\prime} \mathrm{S}$ in the west.

\section{Grania levis Coates \& Erséus, 1985}

Grania levis Coates \& Erséus, 1985: 111-112, fig. 6.

Grania levis - Diaz et al. 1987: tabs 1, 4. — Locke \& Coates 2000: 626.

?Grania cf. levis - Prantoni et al. 2016: 502. 
Type material

\section{Holotype}

UNITED STATES: Georges Bank (USNM 96509).

Paratypes

UNITED STATES: Georges Bank (USNM 96510); off New Jersey (USNM 96511).

\section{Other material}

UNITED STATES: off North Carolina (USNM 1283176; immature specimen, see Prantoni et al. 2016).

\section{Type locality}

UNITED STATES: Georges Bank, SE of Massachusetts, NW Atlantic Ocean.

\section{Habitat}

Intertidal, subtidal to $79 \mathrm{~m}$ (probably to $492 \mathrm{~m}$, see Prantoni et al. 2016), medium to coarse sand.

\section{Distribution}

Georges Bank SE of Massachusetts, on the continental shelf (and slope?) off New Jersey and North Carolina, USA.

\section{Grania reducta Coates \& Erséus, 1985}

Grania reducta Coates \& Erséus, 1985: 110-111, fig. 5.

Grania reducta - Diaz et al. 1987: tabs 3-4. — Locke \& Coates 2000: 626, 628.

\section{Type material}

\section{Holotype}

UNITED STATES: off Maryland (USNM 96512).

\section{Paratype}

UNITED STATES: off Maryland (USNM 96513).

\section{Type locality}

UNITED STATES: off Maryland.

\section{Habitat}

Intertidal, subtidal to $65 \mathrm{~m}$, medium to coarse sand.

\section{Distribution}

Continental shelf off New Jersey, Maryland and Delaware, USA.

\section{Grania ascophora Coates, 1990}

Grania ascophora Coates, 1990: 23-25, fig. 5.

\section{Type material}

\section{Holotype}

AUSTRALIA: Western Australia, Baker Bay (WAM 69.89).

\section{Paratype}

AUSTRALIA: Western Australia, Baker Bay (ROMIZ I2880). 
Type locality

AUSTRALIA: Baker Bay, King George Sound, Western Australia.

\section{Habitat}

Subtidal, $4 \mathrm{~m}$, fine sand with shells and seagrass.

\section{Distribution}

Barker Bay and Princess Royal Harbour, Albany area, Western Australia.

\section{Grania bykane Coates, 1990}

Grania bykane Coates, 1990: 21-23, figs 2, 4a-d.

Grania bykane - Rota et al. 2007: 1001-1004, figs 1a-g, 2a.

\section{Type material}

\section{Holotype}

AUSTRALIA: Western Australia, Princess Royal Harbour (WAM 55.8).

\section{Paratype}

AUSTRALIA: Western Australia, Princess Royal Harbour (WAM 56.89).

\section{Type locality}

AUSTRALIA: Princess Royal Harbour, Albany area, Western Australia.

\section{Habitat}

Intertidal, subtidal to at least $6 \mathrm{~m}$, fine to coarse sand and in sediments under boulders and in seagrass beds.

\section{Distribution}

Southern coast of Western Australia (Albany and Esperance areas), Australia.

\section{Grania crassiducta Coates, 1990}

Grania crassiducta Coates, 1990: 20-21, figs 2, 3a-d.

Grania crassiducta - Coates \& Stacey 1993: 404-406, fig. 9a-f. — Rota et al. 2007: 1004-1006, figs $2 \mathrm{~b}, 3 \mathrm{a}-\mathrm{f}$.

\section{Type material}

\section{Holotype}

AUSTRALIA: Western Australia, Princess Royal Harbour (WAM 51.89).

\section{Paratypes}

AUSTRALIA: Western Australia, Princess Royal Harbour (WAM 53.89, ROMIZ I1279).

\section{Type locality}

AUSTRALIA: Princess Royal Harbour, Albany area, Western Australia.

\section{Habitat}

Intertidal, muddy coarse sand, gravel and mixed sand with pebbles and coral. 


\section{Distribution}

Southern (Albany and Esperance areas) and western (Rottnest Island) coasts of Western Australia.

25. Grania ersei Coates, 1990

Grania ersei Coates, 1990: 17-20, figs 1a-d, 2.

Grania ersei - Coates \& Stacey 1993: 406-408, figs 10a-f. — Rota et al. 2007: 1008-1011, figs 4d-g, 5 a.

Type material

Holotype

AUSTRALIA: Western Australia, Princess Royal Harbour (WAM 61.89).

Paratypes

AUSTRALIA: Western Australia, Princess Royal Harbour (WAM 62.68-68.89, ROMIZ I1273-I1276).

\section{Type locality}

AUSTRALIA: Princess Royal Harbour, Albany area, Western Australia.

\section{Habitat}

Intertidal, subtidal to $26 \mathrm{~m}$, sand among boulders and pebbles, and with algal debris.

\section{Distribution}

South (Albany, Esperance) and west (Rottnest Island) coasts of Western Australia.

26. Grania hastula Coates, 1990

Grania hastula Coates, 1990: 26-28, fig. 7.

\section{Type material}

\section{Holotype}

AUSTRALIA: Western Australia, Middleton Beach (USNM 120714).

\section{Type locality}

AUSTRALIA: Middleton Beach, Albany area, Western Australia.

\section{Habitat}

Intertidal, sand among rocks in algal wash.

\section{Distribution}

Only known from the type locality.

27. Grania hyperoadenia Coates, 1990

Grania hyperoadenia Coates, 1990: 25-26, fig. 6.

Grania hyperoadenia - De Wit et al. 2009: 30-31, fig. 9.

\section{Type material}

Holotype

AUSTRALIA: Western Australia, Baker Bay (WAM 54.85). 


\section{Other material}

AUSTRALIA: Queensland, Lizard Island (AMS W.35560).

Type locality

AUSTRALIA: Baker Bay, King George Sound, Western Australia.

\section{Habitat}

Subtidal, $1.5-4 \mathrm{~m}$ in sand.

\section{Distribution}

Albany area, Western Australia and Lizard Island, Great Barrier Reef, Queensland, Australia.

28. Grania hongkongensis Erséus, 1990

Grania hongkongensis Erséus, 1990: 311-12, fig. 22.

Type material

\section{Holotype}

CHINA: Hong Kong (New Territories), Crooked Island (BMNH 1987.3.39).

\section{Paratypes}

CHINA: Hong Kong (New Territories), Crooked Island (BMNH 1987.3.40, SMNH 3717).

Type locality

CHINA: Mirs Bay, Crooked Island, Hong Kong (New Territories).

\section{Habitat}

Intertidal, subtidal to $15 \mathrm{~m}$, shelly sand.

\section{Distribution}

Only known from the type locality.

\section{Grania inermis Erséus, 1990}

Grania inermis Erséus, 1990: 314-315, fig. 24.

\section{Type material}

\section{Holotype}

CHINA: Hong Kong (New Territories), Crooked Island (BMNH 1987.3.42).

\section{Paratypes}

CHINA: Hong Kong (New Territories), Crooked Island (BMNH 1987.3.43, SMNH 3719).

\section{Type locality}

CHINA: Mirs Bay, Crooked Island, Hong Kong (New Territories).

\section{Habitat}

Shelly sand, 7-14 m.

\section{Distribution}

Only known from the type locality. 
30. Grania stilifera Erséus, 1990

Grania stilifera Erséus, 1990: 312-314, fig. 23.

Type material

Holotype

CHINA: Hong Kong (New Territories), Crooked Island (BMNH 1987.3.41).

Paratype

CHINA: Hong Kong (New Territories), Crooked Island (SMNH 3718).

Type locality

CHINA: Mirs Bay, Crooked Island, Hong Kong (New Territories).

\section{Habitat}

Subtidal, 5-8 m, shelly sand.

\section{Distribution}

Only known from the type locality.

\section{Grania alliata Coates \& Stacey, 1993}

Grania alliata Coates \& Stacey, 1993: 397-399, figs 3-4.

\section{Type material}

\section{Holotype}

AUSTRALIA: Western Australia, Rottnest Island (WAM 192-92).

\section{Type locality}

AUSTRALIA: Rottnest Island, Western Australia.

\section{Habitat}

Intertidal, gravelly sand.

\section{Distribution}

Only known from the type locality.

32. Grania conjuncta Coates \& Stacey, 1993

Grania conjuncta Coates \& Stacey, 1993: 402-404, figs 7-8.

\section{Type material}

\section{Holotype}

AUSTRALIA: Western Australia, Rottnest Island (WAM 193-92).

\section{Type locality}

AUSTRALIA: Rottnest Island, Western Australia.

\section{Habitat}

Subtidal, $2 \mathrm{~m}$, medium to coarse sand. 


\section{Distribution}

Only known from the type locality.

33. Grania longistyla Coates \& Stacey, 1993

Grania longistyla Coates \& Stacey, 1993: 394-397, figs 1-2.

Type material

\section{Holotype}

AUSTRALIA: Western Australia, Rottnest Island (WAM 194-92).

\section{Paratype}

AUSTRALIA: Western Australia, Rottnest Island (WAM 195-92).

\section{Type locality}

AUSTRALIA: Rottnest Island, Western Australia.

\section{Habitat}

Intertidal, gravelly sand.

\section{Distribution}

Only known from the type locality.

\section{Grania vacivasa Coates \& Stacey, 1993}

Grania vacivasa Coates \& Stacey, 1993: 400-402, figs 5-6.

Grania vacivasa - Rota et al. 2007: 1018-1020, figs 8c, 9a-e.

\section{Type material}

\section{Holotype}

AUSTRALIA: Western Australia, Rottnest Island (WAM 196-92).

\section{Paratype}

AUSTRALIA: Western Australia, Rottnest Island (WAM 197-92).

\section{Type locality}

AUSTRALIA: Rottnest Island, Western Australia.

\section{Habitat}

Subtidal, $1 \mathrm{~m}$, in coarse sand.

\section{Distribution}

South (Esperance) and west (Rottnest Island) coasts of Western Australia.

35. Grania acanthochaeta Rota \& Erséus, 1996

Grania acanthochaeta Rota \& Erséus, 1996: 174-175, fig. 4, tab. 1.

\section{Type material}

\section{Holotype}

ANTARCTICA: Ross Island (USNM 172142). 


\section{Paratype}

ANTARCTICA: Ross Island (USNM 172193).

\section{Other material}

ANTARCTICA: Ross Island (USNM 172194-172397).

\section{Type locality}

ANTARCTICA: Ross Island.

\section{Habitat}

Subtidal, $38 \mathrm{~m}$, greyish brown gravelly mud with sponge spicules and valves of Limatula.

\section{Distribution}

Only known from the type locality.

\section{Grania algida Rota \& Erséus, 1996}

Grania algida Rota \& Erséus, 1996: 179-181, fig. 8, tab. 1.

\section{Type material}

\section{Holotype}

ANTARCTICA: Ross Island (USNM 172398).

\section{Paratype}

ANTARCTICA: Ross Island (MZR Oligochaeta 0065).

\section{Type locality}

ANTARCTICA: Ross Island.

\section{Habitat}

Subtidal, 14-40 m, volcanic gravel and cobble.

\section{Distribution}

Only known from the type locality.

\section{Grania angustinasus Rota \& Erséus, 1996}

Grania angustinasus Rota \& Erséus, 1996: 177-178, figs 3b, 6, tab. 1.

\section{Type material}

\section{Holotype}

ANTARCTICA: Ross Island (MCZR Oligochaeta 0059).

\section{Paratypes}

ANTARCTICA: Ross Island (MCZR 0060-0063, SMNH 4759-4761).

\section{Type locality}

ANTARCTICA: Ross Island.

\section{Habitat}

Subtidal, 35-126 m, fine sand. 


\section{Distribution}

Only known from the type locality.

38. Grania antarctica Rota \& Erséus, 1996

Grania antarctica Rota \& Erséus, 1996: 178-179, figs 3c, 7, tab. 1.

Type material

Holotype

ANTARCTICA: Ross Island (USNM 172400).

Paratype

ANTARCTICA: Ross Island (USNM 172402, MCZR Oligochaeta 0059).

Type locality

ANTARCTICA: Ross Island.

\section{Habitat}

Subtidal, 14-31 m, volcanic gravel and cobble.

\section{Distribution}

Only known from the type locality.

39. Grania carchinii Rota \& Erséus, 1996

Grania carchinii Rota \& Erséus, 1996: 175-177, fig. 5, tab. 1.

Type material

Holotype

ANTARCTICA: Ross Island (MCZR Oligochaeta 0057).

Paratype

ANTARCTICA: Ross Island (MCZR Oligochaeta 0058).

Type locality

ANTARCTICA: Ross Island.

\section{Habitat}

Subtidal, $35 \mathrm{~m}$, fine sand with mica shale, shell debris and some pebbles.

\section{Distribution}

Only known from the type locality.

40. Grania hirsuticauda Rota \& Erséus, 1996

Grania hirsuticauda Rota \& Erséus, 1996: 175-177, fig. 5, tab. 1.

Type material

Holotype

ANTARCTICA: Ross Island (USNM 172136).

Paratypes

ANTARCTICA: Ross Island (USNM 172137-172138). 
Type locality

ANTARCTICA: Ross Island.

\section{Habitat}

Subtidal, 5-585 m, volcanic gravel, sandy mud, small rocks, ectoproct and sponge debris.

\section{Distribution}

Only known from the type locality.

\section{Grania darwinensis (Coates \& Stacey, 1997)}

Randidrilus darwinensis Coates \& Stacey, 1997: 70-72, fig. 1.

Grania darwinensis - Rota et al. 2003: 504-509, fig. 3.

\section{Type material}

\section{Holotype}

AUSTRALIA: Northern Territory, Darwin (NTM Wo 0084).

\section{Paratypes}

AUSTRALIA: Northern Territory, Darwin (NTM Wo 0085-0087, ROMIZ I2457-I2458).

\section{Type locality}

AUSTRALIA: Darwin, Northern Territory.

\section{Habitat}

Intertidal, subtidal to $16 \mathrm{~m}$, medium to coarse sand, clay or silty sediments.

\section{Distribution}

Northern Territory and Western Australia.

\section{Grania eurystila Coates \& Stacey, 1997}

Grania eurystila Coates \& Stacey, 1997: 73-74, fig. 2.

\section{Type material}

\section{Holotype}

AUSTRALIA: Northern Territory, Darwin (NTM Wo 0081).

Paratypes

AUSTRALIA: Northern Territory, Darwin (NTM Wo 0082-0083, ROMIZ I2479).

\section{Type locality}

AUSTRALIA: Darwin, Northern Territory.

\section{Habitat}

Intertidal rockpool.

\section{Distribution}

Only known from two locations in the inner part of Darwin Harbour, Northern Territory, Australia. 
43. Grania integra Coates \& Stacey, 1997

Grania integra Coates \& Stacey, 1997: 74-76, fig. 3.

Grania integra - Rota et al. 2003: 499-501, fig. 1.

\section{Type material}

\section{Holotype}

AUSTRALIA: Northern Territory, Darwin (NTM Wo 0079).

Paratype

AUSTRALIA: Northern Territory, Darwin (NTM Wo 0080).

\section{Type locality}

AUSTRALIA: Darwin, Northern Territory.

\section{Habitat}

Intertidal crevices with sand gravel, pebbles and heterogeneous sediments.

\section{Distribution}

Darwin Harbour, Northern Territory and Nickol Bay, Dampier area, Western Australia.

44. Grania lasserrei Rota \& Erséus, 1997

Grania lasserrei Rota \& Erséus, 1997: 34-37, fig. 3, tab.1.

Enchytraeus monochaetus - Michaelsen 1888: 66, figs 6A-C; part., pl. 2.

Michaelsena monochaeta - Michaelsen 1921: 3, part.

Grania monochaeta - Erséus \& Lasserre 1977: 299-300, figs 1A-D.

\section{Type material}

\section{Holotype}

UNITED KINGDOM: South Georgia (SMNH 4803).

\section{Paratypes}

UNITED KINGDOM: South Georgia (SMNH 48044806, MZUT 1078, BMNH 1996:916).

\section{Type locality}

UNITED KINGDOM: South Georgia, SW Atlantic Ocean.

\section{Habitat}

Intertidal, subtidal to $20 \mathrm{~m}$.

\section{Distribution}

Only known from the type locality.

45. Grania stephensoniana Rota \& Erséus, 1997

Grania stephensoniana Rota \& Erséus, 1997: 37-39, figs 4-5, tab. 1.

Michaelsena monochaeta - Stephenson 1932: 263, fig. 14. 
Type material

Holotype

UNITED KINGDOM: South Georgia (BMNH 1931.6.23.78).

Paratypes

UNITED KINGDOM: South Georgia (BMNH 1933.2.23.946-1933.2.23.948).

Type locality

UNITED KINGDOM: South Georgia, SW Atlantic Ocean.

\section{Habitat}

$60 \mathrm{~m}$, rocky bottom.

\section{Distribution}

Only known from the type locality.

\section{Grania mira Locke \& Coates, 1998}

Grania mira Locke \& Coates, 1998: 1103-1107, figs 1-5.

Grania sp. - Healy 1996a: 53, 56-57, fig. 1, tabs 1-2; 1996b: 1287.

\section{Type material}

\section{Holotype}

IRELAND: Carnsore Point (NMI 4.1998).

\section{Paratypes}

IRELAND: Carnsore Point (NMI 5.1998-6.1998).

\section{Type locality}

IRELAND: Carnsore Point, County Wexford.

\section{Habitat}

Intertidal, sediments trapped in dense turf of Corallina officinalis on horizontal or gently sloping rock.

\section{Distribution}

Only known from the type locality.

47. Grania hylae Locke \& Coates, 1999

Grania hylae Locke \& Coates, 1999: 605-609, figs 6-7, 11, tab. 1.

Grania hylae - Locke \& Coates 2000: 619-621, 626, fig. 4b.

\section{Type material}

\section{Holotype}

BERMUDA: Paget Island (USNM 185954).

Paratypes

UNITED STATES: Florida, Fowey Rocks (USNM 185955).

BERMUDA: Castle Island (USNM 185956, BAMZ 199180 007). 
Type locality

BERMUDA: Paget Island.

\section{Habitat}

Intertidal, subtidal to $17 \mathrm{~m}$, medium to coarse sand with rocks.

\section{Distribution}

Rocky Hill Park, Castle Island and Paget Island, Bermuda, and Fowey Rocks, Miami, Florida, USA.

48. Grania laxartus Locke \& Coates, 1999

Grania laxartus Locke \& Coates, 1999: 602-605, figs 2-6, 11, tab. 1.

Grania laxartus - Locke \& Coates 2000: 619-621, 626-627, figs 4c, 6a.

\section{Type material}

\section{Holotype}

BERMUDA: Ferry Point Bridge (USNM 185951).

\section{Paratypes}

BERMUDA: Ferry Reach (USNM 185952, BAMZ 199180 006).

BELIZE: Carrie Bow Cay (USNM 185953).

\section{Type locality}

BERMUDA: Ferry Point Bridge.

\section{Habitat}

Intertidal pools with accumulation of sand and fine to medium-coarse calcareous sand.

\section{Distribution}

Ferry Point Bridge, Whalebone Bay, Pearl Island, Ferry Reach and Smith's Sound, Bermuda, and Carrie Bow Cay, Belize.

49. Grania dolichura Rota \& Erséus, 2000

Grania dolichura Rota \& Erséus, 2000: 249-252, fig. 3.

Grania dolichura - Rota et al. 2007: 1006-1008, figs 4a-c.

\section{Type material}

\section{Holotype}

AUSTRALIA: Tasmania, Little Musselroe (QVM 14: 3889).

\section{Paratypes}

AUSTRALIA: Tasmania, Little Musselroe (QVM 14:3890-14:3897, SMNH 5203-5206, MCZR Oligochaeta 0085-0088).

\section{Type locality}

AUSTRALIA: Little Musselroe, Tasmania. 


\section{Habitat}

Intertidal, silt-clay sediments.

\section{Distribution}

Widespread around Tasmania, Australia.

50. Grania tasmaniae Rota \& Erséus, 2000

Grania tasmaniae Rota \& Erséus, 2000: 247-249, fig. 2.

\section{Type material}

\section{Holotype}

AUSTRALIA: Tasmania, Low Head (QVM 14: 3887).

\section{Paratype}

AUSTRALIA: Tasmania, Low Head (QVM 14: 3888).

\section{Type locality}

AUSTRALIA: Low Head, Tamar Estuary, Tasmania.

\section{Habitat}

Intertidal, silt-clay sediments.

\section{Distribution}

Only known from the type locality.

51. Grania aquitana Rota \& Erséus, 2003

Grania aquitana Rota \& Erséus, 2003: 226-229, fig. 7, tab. 1.

\section{Type material}

\section{Holotype}

FRANCE: Bassin d'Arcachon (SMNH 5729).

\section{Paratypes}

FRANCE: Bassin d'Arcachon (SMNH 5730-5733).

\section{Type locality}

FRANCE: Bassin d'Arcachon.

\section{Habitat}

Subtidal, 2-5 m, fine sand.

\section{Distribution}

Only known from the type locality.

52. Grania canaria Rota \& Erséus, 2003

Grania canaria Rota \& Erséus, 2003: 213-215, fig. 1, tab. 1. 
Type material

\section{Holotype}

SPAIN: Tenerife (ZMA V.OL 9344).

Paratypes

SPAIN: Tenerife (SMNH 5710-5711).

\section{Type locality}

SPAIN: Tenerife, Canary Islands.

\section{Habitat}

Intertidal, sand and gravel.

\section{Distribution}

Only known from the type locality.

53. Grania fortunata Rota \& Erséus, 2003

Grania fortunata Rota \& Erséus, 2003: 215-218, fig. 2, tab. 1.

\section{Type material}

\section{Holotype}

SPAIN: Tenerife (SMNH 5712).

\section{Paratype}

SPAIN: Tenerife (SMNH 5713, MCZR Oligochaeta 0103-0104).

\section{Type locality}

SPAIN: Tenerife, Canary Islands.

\section{Habitat}

Subtidal, 12-17 m, fine and muddy sands associated with beds of the seagrass Cymodocea nodosa.

\section{Distribution}

Only known from the type locality.

\section{Grania mauretanica Rota \& Erséus, 2003}

Grania mauretanica Rota \& Erséus, 2003: 224-226, fig. 6, tab. 1.

\section{Type material}

\section{Holotype}

MOROCCO: off Casablanca (SMNH 5718).

\section{Paratypes}

MOROCCO: off Casablanca (SMNH 5719-5720, MCZR Oligochaeta 0107).

Type locality

MOROCCO: off Casablanca. 


\section{Habitat}

Subtidal, 173 m, mud with shell debris.

\section{Distribution}

Only known from the type locality.

55. Grania papillinasus Rota \& Erséus, 2003

Grania papillinasus Rota \& Erséus, 2003: 239-240, fig. 13.

\section{Type material}

\section{Holotype}

FRANCE: Gulf of Gascogne (SMNH 5726).

\section{Paratypes}

FRANCE: Gulf of Gascogne (SMNH 5727-5728, MCZR Oligochaeta 0124-0126).

\section{Type locality}

FRANCE: Gulf of Gascogne, lower continental slope.

\section{Habitat}

Deep sea, 2630-2885 m, most likely very fine sediments.

\section{Distribution}

Gulf of Gascogne, off France and off the eastern USA (i.e., both sides of the North Atlantic) (see Erséus \& Rota 2003).

\section{Grania torosa Rota \& Erséus, 2003}

Grania torosa Rota \& Erséus, 2003: 237-239, fig. 12.

\section{Type material}

\section{Holotype}

NE ATLANTIC OCEAN: Rockall Trough (SMNH 5721).

\section{Paratypes}

NE ATLANTIC OCEAN: Rockall Trough (SMNH 5722-5725, MCZR Oligochaeta 0123).

\section{Type locality}

NE ATLANTIC OCEAN: Rockall Trough, off Scotland.

\section{Habitat}

Continental slope, 1170-1800 m, fine sandy and hemi-pelagic ooze.

\section{Distribution}

Northern Rockall Trough, off the coast of Scotland, to near the entrance to the English Channel (NE Atlantic Ocean).

\section{Grania vikinga Rota \& Erséus, 2003}

Grania vikinga Rota \& Erséus, 2003: 222-224, fig. 5. 
Grania roscoffensis (part) - sensu Erséus 1977: 294, tab. 1.

Grania vikinga - van Haaren 2016: 144-145, figs 22, 24, 27-28, 59.

\section{Type material}

\section{Holotype}

SWEDEN: Skagerrak (SMNH 5714).

Paratypes

SWEDEN: Skagerrak (SMNH 5716-5717, MCZR Oligochaeta 0105-0106).

Type locality

SWEDEN: Skagerrak, Bohuslän.

\section{Habitat}

Subtidal, 10-46 m, sand.

\section{Distribution}

West coast of Sweden, the Netherlands.

58. Grania ocarina Rota, Erséus \& Wang, 2003

Grania ocarina Rota, Erséus \& Wang, 2003: 502-504, fig. 2.

\section{Type material}

\section{Holotype}

AUSTRALIA: Western Australia, Withnell Bay (WAM V 4351).

Paratype

AUSTRALIA: Western Australia, Withnell Bay (WAM V 4352, SMNH 5868, MCZR Oligochaeta 0128).

\section{Type locality}

AUSTRALIA: Withnell Bay, Dampier Area, Western Australia.

\section{Habitat}

Barely subtidal, $0.5 \mathrm{~m}$, medium to coarse sand.

\section{Distribution}

Only known from the type locality.

59. Grania cinctura De Wit \& Erséus, 2007

Grania cinctura De Wit \& Erséus, 2007: 33-36, fig. 3, tab. 1.

\section{Type material}

\section{Holotype}

FRANCE: New Caledonia, Lifou (SMNH 6559).

Paratypes

FRANCE: New Caledonia, Lifou (SMNH 6560-6564); New Caledonia, Touho (SMNH 6565-6568, 6572). 
Type locality

FRANCE: Lifou, Loyalty Islands, New Caledonia.

\section{Habitat}

Intertidal, subtidal to $6 \mathrm{~m}$, fine to coarse sand.

\section{Distribution}

Touho and Nouméa areas, and Loyalty Islands, New Caledonia.

60. Grania curta De Wit \& Erséus, 2007

Grania curta De Wit \& Erséus, 2007: 38-40, fig. 5, tab. 1.

\section{Type material}

\section{Holotype}

FRANCE: New Caledonia, Lifou (SMNH 6583).

\section{Paratypes}

FRANCE: New Caledonia, Lifou (SMNH 6584-6588).

Type locality

FRANCE: Lifou, Loyalty Islands, New Caledonia.

\section{Habitat}

Barely subtidal, $0.5 \mathrm{~m}$, heterogeneous sand.

\section{Distribution}

Only known from the type locality.

\section{Grania fiscellata De Wit \& Erséus, 2007}

Grania fiscellata De Wit \& Erséus, 2007: 45-47, fig. 9, tab. 1.

\section{Type material}

\section{Holotype}

FRANCE: New Caledonia, Touho (SMNH 6610).

\section{Paratypes}

FRANCE: New Caledonia, Touho (SMNH 6611-6613); New Caledonia, Lifou (SMNH 6617).

\section{Type locality}

FRANCE: Touho, Loyalty Islands, New Caledonia.

\section{Habitat}

Intertidal, subtidal to $3 \mathrm{~m}$, heterogeneous sand.

\section{Distribution}

Touho area and Lifou (Loyalty Islands), New Caledonia. 
62. Grania fustata De Wit \& Erséus, 2007

Grania fustata De Wit \& Erséus, 2007: 40-42, fig. 6, tab. 1.

Type material

Holotype

FRANCE: New Caledonia, Touho (SMNH 6589).

Paratypes

FRANCE: New Caledonia, Touho (SMNH 6590-6598).

Type locality

FRANCE: Touho, Loyalty Islands, New Caledonia.

\section{Habitat}

Intertidal and barely subtidal, coarse sand and gravel.

\section{Distribution}

Only known from the type locality.

63. Grania galbina De Wit \& Erséus, 2007

Grania galbina De Wit \& Erséus, 2007: 36-38, fig. 4, tab. 1.

\section{Type material}

\section{Holotype}

FRANCE: New Caledonia, Lifou (SMNH 6573).

Paratypes

FRANCE: New Caledonia, Lifou (SMNH 6574-6582).

\section{Type locality}

FRANCE: Lifou, Loyalty Islands, New Caledonia.

\section{Habitat}

Intertidal, subtidal to $13 \mathrm{~m}$, heterogeneous sand.

\section{Distribution}

Nouméa area and Lifou (Loyalty Islands), New Caledonia.

64. Grania novacaledonia De Wit \& Erséus, 2007

Grania novacaledonia De Wit \& Erséus, 2007: 31-33, fig. 2, tab. 1.

Type material

\section{Holotype}

FRANCE: New Caledonia, Touho (SMNH 6549).

\section{Paratypes}

FRANCE: New Caledonia, Touho (SMNH 6550-6558). 
Type locality

FRANCE: Touho, Loyalty Islands, New Caledonia.

\section{Habitat}

Intertidal, subtidal to $21 \mathrm{~m}$, heterogeneous sand.

\section{Distribution}

Touho and Nouméa areas, New Caledonia.

\section{Grania papillata De Wit \& Erséus, 2007}

Grania papillata De Wit \& Erséus, 2007: 42-45, figs 7-8, tab. 1.

\section{Type material}

\section{Holotype}

FRANCE: New Caledonia, Lifou (SMNH 6599).

\section{Paratypes}

FRANCE: New Caledonia, Lifou (SMNH 6600-6602); New Caledonia, Touho (SMNH 6603-6609).

\section{Type locality}

FRANCE: Lifou, Loyalty Islands, New Caledonia.

\section{Habitat}

Intertidal, subtidal to $22 \mathrm{~m}$, heterogeneous sand.

\section{Distribution}

Touho area and Lifou (Loyalty Islands), New Caledonia.

\section{Grania quaerens Rota, Wang \& Erséus, 2007}

Grania quaerens Rota, Wang \& Erséus, 2007: 1011-1013, figs 5b-d, 6a-i.

\section{Type material}

\section{Holotype}

AUSTRALIA: Western Australia, New Island (WAM V 7315).

\section{Paratypes}

AUSTRALIA: Western Australia, New Island (WAM V 7316-7319, SMNH 6803-6808, MCZR Oligochaeta 0146-0149).

\section{Type locality}

AUSTRALIA: New Island, Western Australia.

\section{Habitat}

Intertidal, medium to coarse sand.

\section{Distribution}

South coast of Western Australia. 
67. Grania sperantia Rota, Wang \& Erséus, 2007

Grania sperantia Rota, Wang \& Erséus, 2007: 1014-1017, figs 7a-h, 8a-b.

Type material

\section{Holotype}

AUSTRALIA: Western Australia, Lucky Bay (WAM V 7320).

Paratypes

AUSTRALIA: Western Australia, Lucky Bay (WAM V 7321-7326, SMNH 6809-6817, MCZR Oligochaeta 0150-0155).

\section{Type locality}

AUSTRALIA: Lucky Bay, Western Australia.

\section{Habitat}

Barely subtidal, 0.5-2 m, medium to coarse sand.

\section{Distribution}

South coast of Western Australia.

68. Grania breviductus De Wit, Rota \& Erséus, 2009

Grania breviductus De Wit, Rota \& Erséus, 2009: 19-21, figs 2, 10a.

Type material

\section{Holotype}

AUSTRALIA: Queensland, Heron Island (AMS W.35536).

Paratypes

AUSTRALIA: Queensland, Heron Island (AMS W.35537-.35542, SMNH 7761-7766).

\section{Type locality}

AUSTRALIA: Heron Island, Great Barrier Reef, Queensland, Australia.

\section{Habitat}

Intertidal, coarse sand.

\section{Distribution}

Only known from the type locality.

69. Grania regina De Wit, Rota \& Erséus, 2009

Grania regina De Wit, Rota \& Erséus, 2009: 21-23, figs 3-4, 10 b.

Type material

\section{Holotype}

AUSTRALIA: Queensland, Heron Island (AMS W.35543).

Type locality

AUSTRALIA: Heron Island, Great Barrier Reef, Queensland, Australia. 


\section{Habitat}

Subtidal, $15 \mathrm{~m}$, fine sand.

\section{Distribution}

Only known from the type locality.

70. Grania homochaeta De Wit, Rota \& Erséus, 2009

Grania homochaeta De Wit, Rota \& Erséus, 2009: 23-25, figs 5, 10c.

\section{Type material}

\section{Holotype}

AUSTRALIA: Queensland, Heron Island (AMS W.35544).

Paratype

AUSTRALIA: Queensland, Heron Island (MNH 7767).

\section{Type locality}

AUSTRALIA: Heron Island, Great Barrier Reef, Queensland, Australia.

\section{Habitat}

Subtidal, $18 \mathrm{~m}$, gravelly fine sand.

\section{Distribution}

Only known from the type locality.

71. Grania colorata De Wit, Rota \& Erséus, 2009

Grania colorata De Wit, Rota \& Erséus, 2009: 25-27, figs 6-7, 10d.

\section{Type material}

\section{Holotype}

AUSTRALIA: Queensland, Heron Island (AMS W.35545).

\section{Paratypes}

AUSTRALIA: Queensland, Heron Island (AMS W.35546-.35553, SMNH 7768-7772).

\section{Type locality}

AUSTRALIA: Heron Island, Great Barrier Reef, Queensland, Australia.

\section{Habitat}

Subtidal, $7 \mathrm{~m}$, in heterogeneous sand.

\section{Distribution}

Only known from the type locality.

72. Grania occulta De Wit \& Erséus, 2010

Grania occulta De Wit \& Erséus, 2010: 287-289, fig. 3. 
Type material

Holotype

SWEDEN: Gullmar Fjord (SMNH 7844).

Type locality

SWEDEN: Gullmar Fjord, Bohuslän.

\section{Habitat}

Subtidal, 10-25 m, shell sand with some mud.

\section{Distribution}

Only known from the type locality.

73. Grania brasiliensis Prantoni, De Wit \& Erséus, 2016

Grania brasiliensis Prantoni, De Wit \& Erséus, 2016: 489-491, fig. 1.

\section{Type material}

\section{Holotype}

BRAZIL: Paraná State, Paranaguá Bay (ZUEC CLI 04).

\section{Paratypes}

BRAZIL: Paraná State, Paranaguá Bay (ZUEC CLI 05); São Paulo State, São Paulo (ZUEC CLI 06-07).

\section{Type locality}

BRAZIL: Paranaguá Bay, Paraná State.

\section{Habitat}

Intertidal, subtidal to $7 \mathrm{~m}$, medium to coarse sand with some mud and lots of mollusc and barnacle shells.

\section{Distribution}

Coasts of Paraná and São Paulo States, Brazil.

74. Grania bekkouchei Prantoni, De Wit \& Erséus, 2016

Grania bekkouchei Prantoni, De Wit \& Erséus, 2016: 491-492, figs 2, 4a-c.

\section{Type material}

\section{Holotype}

SOUTH AFRICA: Western Cape (SAMC A82466).

\section{Type locality}

SOUTH AFRICA: Western Cape.

\section{Habitat}

Intertidal, coarse sand in rock crevice.

\section{Distribution}

Only known from the type locality. 
75. Grania cryptica Prantoni, De Wit \& Erséus 2016

Grania cryptica Prantoni, De Wit \& Erséus, 2016: 492-493, figs 3, 4d-f.

Type material

Holotype

SOUTH AFRICA: Western Cape (SAMC A82473).

Type locality

SOUTH AFRICA: Western Cape.

\section{Habitat}

Lower intertidal, rockpool.

\section{Distribution}

Only known from the type locality.

76. Grania capensis Prantoni, De Wit \& Erséus, 2016

Grania capensis Prantoni, De Wit \& Erséus, 2016: 493-495, fig. 5.

Type material

\section{Holotype}

SOUTH AFRICA: Western Cape (SAMC A82474).

\section{Paratype}

SOUTH AFRICA: Western Cape (SAMC A82475).

Type locality

SOUTH AFRICA: Western Cape.

\section{Habitat}

Lower intertidal, rockpool.

\section{Distribution}

Only known from the type locality.

77. Grania simonae Prantoni, De Wit \& Erséus, 2016

Grania simonae Prantoni, De Wit \& Erséus, 2016: 495-497, fig. 6.

Type material

Holotype

SOUTH AFRICA: Western Cape (SAMC A82476).

Paratypes

SOUTH AFRICA: Western Cape (SAMC 82477-82482).

Type locality

SOUTH AFRICA: Western Cape. 


\section{Habitat}

Intertidal, crevice between rocks.

\section{Distribution}

Only known from the type locality.

78. Grania hinojosai Prantoni, De Wit \& Erséus, 2016

Grania hinojosai Prantoni, De Wit \& Erséus, 2016: 497-498, fig. 7.

Grania sp. Chile 1- De Wit et al. 2011: 513.

\section{Type material}

\section{Holotype}

CHILE: Coquimbo (ZUEC CLI 08).

\section{Paratypes}

CHILE: Coquimbo (ZUEC CLI 09-12).

\section{Type locality}

CHILE: Coquimbo, Elqui.

\section{Habitat}

Intertidal, sand among rocks.

\section{Distribution}

Puerto Aldea to Pampilla Point, Coquimbo, Elqui, Chile.

79. Grania chilensis Prantoni, De Wit \& Erséus, 2016

Grania chilensis Prantoni, De Wit \& Erséus, 2016: 498-500, fig. 8.

Grania sp. Chile 2-De Wit et al. 2011: 513, 517.

\section{Type material}

\section{Holotype}

CHILE: Valdivia (ZUEC CLI 13).

\section{Paratypes}

CHILE: Valdivia (ZEUC CLI 14-19).

\section{Type locality}

CHILE: Valdivia.

\section{Habitat}

Intertidal, sand among rocks and heterogeneous sand with organic material.

\section{Distribution}

Along coast of Chile, from about $30.3^{\circ}$ to $39.8^{\circ} \mathrm{S}$. 
80. Grania unitheca Prantoni, De Wit \& Erséus, 2016

Grania unitheca Prantoni, De Wit \& Erséus, 2016: 500-501, fig. 9.

\section{Type material}

\section{Holotype}

UNITED STATES: off North Carolina (USNM 1283175).

\section{Type locality}

UNITED STATES: off North Carolina.

\section{Habitat}

Subtidal, $17 \mathrm{~m}$, sand.

\section{Distribution}

Only known from the type locality.

\section{Grania carolinensis Prantoni, De Wit \& Erséus, 2016}

Grania carolinensis Prantoni, De Wit \& Erséus, 2016: 501-502, fig. 10.

\section{Type material}

\section{Holotype}

UNITED STATES: off North Carolina (USNM 1283174).

\section{Type locality}

UNITED STATES: off North Carolina.

\section{Habitat}

Continental shelf slope, $492 \mathrm{~m}$, sand.

\section{Distribution}

Only known from the type locality.

\section{Discussion}

Despite the many species of Grania described from the Australian continent and a few other southern regions of the globe (e.g., New Caledonia and Antarctica, see Fig. 2), taxa from the African and South American continents have been completely ignored until recently. This situation changed with the seven new species recently described from Brazil (1), Chile (2) and South Africa (4 spp.) (Prantoni et al. 2016).

The geographical distribution of the various species of Grania is strongly concordant with the phylogeny of the genus, as estimated by analyses of molecular data (De Wit et al. 2011; Prantoni et al. 2016). Most of the individual species appear to be endemic to rather small geographical areas, which suggests a limited capability of dispersion. However, as a whole, the genus is divided into at least three distinct evolutionary lineages, each with a broad, but geographically coherent distribution in the world; this is based on a sample of 28 genetically analyzed species (Prantoni et al. 2016). The first lineage (green numbers on Fig. 2) comprises species from the Atlantic Ocean (including a sublineage of four species from South Africa). The second (blue numbers) are species from Australia and southern Asia (Hong 
Kong), and the third are species from the South Pacific and Atlantic regions (red numbers). An interesting aspect is the phylogenetic placement of G. americana in the North Atlantic and G. brasiliensis in the South Atlantic. These two species belong to an otherwise Pacific group (the third lineage), and it is suggested that they share a common ancestor that migrated from the Pacific region before the closing of the Isthmus of Panama 3 Ma (De Wit et al. 2011; Prantoni et al. 2016). However, as most Neotropical coasts remain unexplored, additional species of Grania from both sides of South America are needed to corroborate (or refute) this hypothesis.

In conclusion, more intense sampling efforts in many parts of the world are crucial to enable further studies of the evolutionary and biogeographical history of Grania. The 81 species described to date is a high number for a marine genus of Enchytraeidae, and yet, this number certainly does not represent the actual diversity of the genus Grania. Overall, our systematic knowledge of marine clitellates (oligochaetes and leeches) is poor, with perhaps only about $10 \%$ of the species diversity known (Appeltans et al. 2012). The lack of specialists around the world is evidently one of the reasons for this (Prantoni et al. 2014), but it is also a threat to any rapid improvement of the situation. As a partial solution, the combined efforts of taxonomists and ecologists may come as a first and necessary step towards a better understanding of the group as well as of marine clitellate species diversity as a whole.

\section{Acknowledgements}

We thank Pierre De Wit for permission to publish his photo of a specimen of Grania chilensis, and Klara Dozsa-Farkas and one anonymous reviewer for good suggestions regarding the final version of the text. This study was supported by CAPES Brazilian Foundation (CAPES-process: BEX 11676/13-2), which provided a $\mathrm{PhD}$ fellowship for the first author. The second author was supported by the Swedish Taxonomy Initiative (ArtDatabanken).

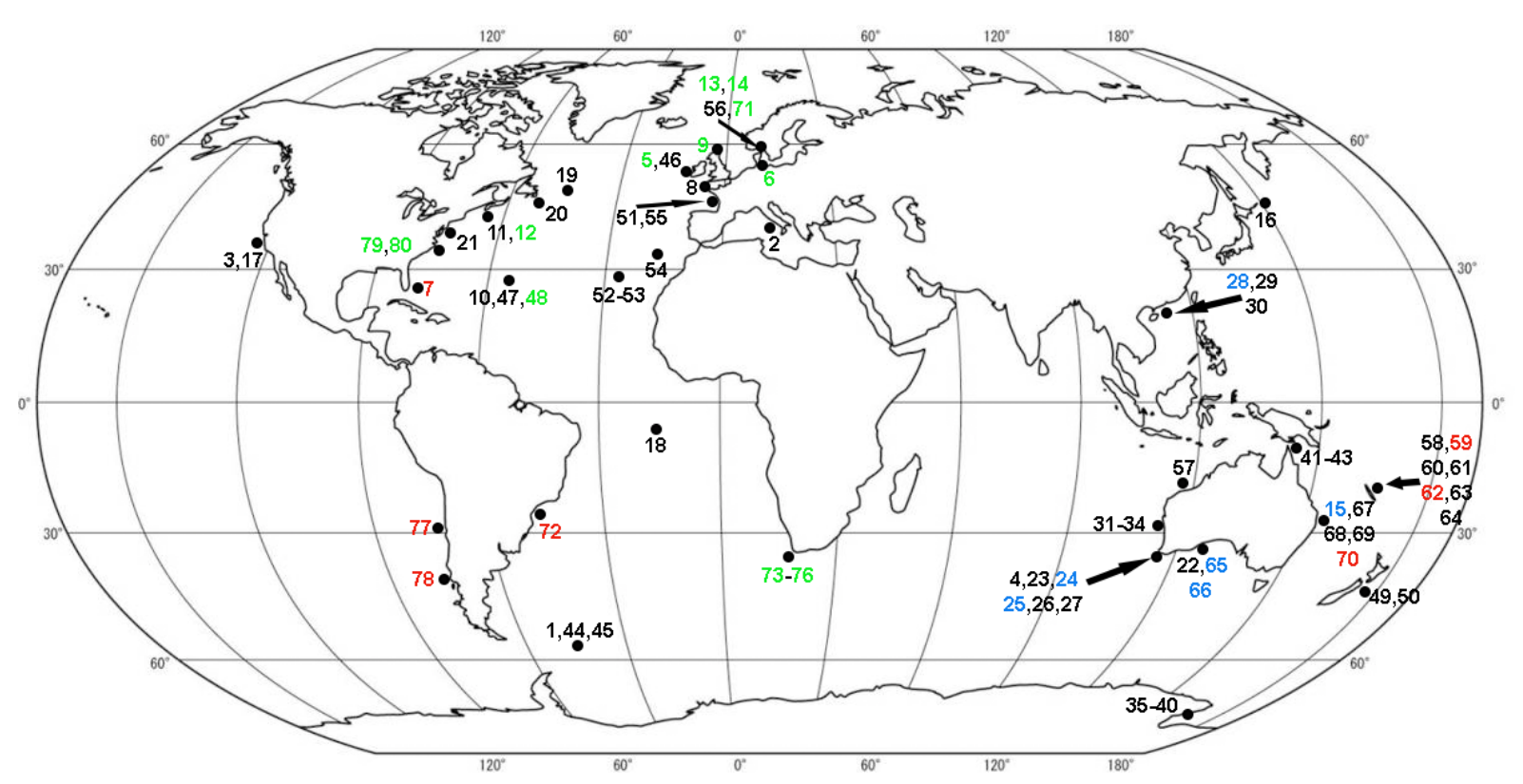

Fig. 2. World map showing the description localities, and the three evolutionary lineages (color-marked) of Grania spp. genetically analyzed by Prantoni et al. (2016: clades A, B and C). Green numbers = Atlantic species (clade A); red numbers = South Pacific and Atlantic species (clade B); blue numbers = Australian and Asian species (clade C); black numbers = species presently without molecular data, i.e., not yet allocated to any particular lineage. 


\section{References}

Appeltans W., Ahyong S.T., Anderson G., Angel M.V., Artois T., Bailly N., Bamber R., Barber A., Bartsch I., Berta A., Błażewicz-Paszkowycz M., Bock P., Boxshall G., Boyko C.B., Brandão S.N., Bray R.A., Bruce N.L., Cairns S.D., Chan T.-Y., Cheng L., Collins A.G., Cribb T., Curini-Galletti M., Dahdouh-Guebas F., Davie P.J.F., Dawson M.N., De Clerck O., Decock W., De Grave S., de Voogd N.J., Domning D.P., Emig C.C., Erséus C., Eschmeyer W., Fauchald K., Fautin D.G., Feist S.W., Fransen C.H.J.M., Furuya H., Garcia-Alvarez O., Gerken S., Gibson D., Gittenberger A., Gofas S., GómezDaglio L., Gordon D.P., Guiry M.D., Hernandez F., Hoeksema B.W., Hopcroft R.R., Jaume D., Kirk P., Koedam N., Koenemann S., Kolb J.B., Kristensen R.M., Kroh A., Lambert G., Lazarus D.B., Lemaitre R., Longshaw M., Lowry J., Macpherson E., Madin L.P., Mah C., Mapstone G., McLaughlin P.A., Mees J., Meland K., Messing C.G., Mills C.E., Molodtsova T.N., Mooi R., Neuhaus B., Ng P.K.L., Nielsen C., Norenburg J., Opresko D.M., Osawa M., Paulay G., Perrin W., Pilger J.F., Poore G.C.B., Pugh P., Read G.B., Reimer J.D., Rius M., Rocha R.M., Saiz-Salinas J.I., Scarabino V., Schierwater B., Schmidt-Rhaesa A., Schnabel K.E., Schotte M., Schuchert P., Schwabe E., Segers H., Self-Sullivan C., Shenkar N., Siegel V., Sterrer W., Stöhr S., Swalla B., Tasker M.L., Thuesen E.V., Timm T., Todaro M.A., Turon X., Tyler S., Uetz P., van der Land J., Vanhoorne B., van Ofwegen L.P., van Soest R.W.M., Vanaverbeke J., Walker-Smith G., Walter T.C., Warren A., Williams G.C., Wilson S.P., Costello M.J. 2012 The magnitude of global marine species diversity. Current Biology 22: 2189-2202.

https://doi.org/10.1016/j.cub.2012.09.036

Beddard F.E. 1895. A Monograph of the Order Oligochaeta. Clarendon Press, Oxford.

Bonomi G. \& Erséus C. 1984. A taxonomic and faunistic survey of the marine Tubificidae and Enchytraeidae (Oligochaeta) of Italy. Introduction and preliminary results. Hydrobiologia 115: 207210. https://doi.org/10.1007/BF00027918

Coates K.A. 1984. Specific criteria in Grania (Oligochaeta, Enchytraeidae). Hydrobiologia 115: 45-50. https://doi.org/10.1007/BF00027891

Coates K.A. 1990. Marine Enchytraeidae (Oligochaeta, Annelida) of the Albany area, Western Australia. In: Wells F., Walker D., Kirkman H. \& Lethbridge R. (eds) Proceedings of the Third International Marine Biological Workshop: The Marine Flora and Fauna of Albany, Western Australia: 13-41. Western Australian Museum, Perth.

Coates K.A. \& Ellis D.V. 1981. Taxonomy and distribution of marine Enchytraeidae (Oligochaeta) in British Columbia. Canadian Journal of Zoology 59: 2129-2150. https://doi.org/10.1139/z81-290

Coates K.A. \& Erséus C. 1980. Two species of Grania (Oligochaeta, Enchytraeidae) from the Pacific Coast of North America. Canadian Journal of Zoology 58: 1037-1041. https://doi.org/10.1139/z80-146

Coates K.A. \& Erséus C. 1985. Marine enchytraeids (Oligochaeta) of the coastal Northwest Atlantic (northern and mid U.S.A.). Zoologica Scripta 14: 103-116.

https://doi.org/10.1111/j.1463-6409.1985.tb00181.x

Coates K.A. \& Stacey D. 1993. The marine Enchytraeidae (Oligochaeta, Annelida) of Rottnest Island, Western Australia. In: Wells F., Walker D., Kirkman H. \& Lethbridge R. (eds) Proceedings of the Fifth International Marine Biological Workshop: The Marine Flora and Fauna of Rottnest Island, Western Australia: 391-414. Western Australian Museum, Perth.

Coates K.A. \& Stacey D. 1997. Enchytraeids (Oligochaeta: Annelida) of the lower shore and shallow subtidal of Darwin Harbour, Northern Territory, Australia. In: Hanley J., Caswell G., Megirian D. \& Larson H. (eds) Proceedings of the Sixth International Marine Biological Workshop: The Marine Flora and Fauna of Darwin Harbour, Northern Territory, Australia: 67-79. Museums and Art Galleries of the Northern Territory and the Australian Marine Sciences Association, Darwin, Australia. 
De Wit P. 2006. Grania maricola. Fauna \& Flora 101 (2): 25.

De Wit P. 2010. Systematics of Grania (Clitellata: Enchytraeidae), an Interstitial Annelid Taxon. PhD Thesis. University of Gothenburg, Sweden.

De Wit P. \& Erséus C. 2007. Seven new species of Grania (Annelida: Clitellata: Enchytraeidae) from New Caledonia, South Pacific Ocean. Zootaxa 1426: 27-50. https://doi.org/10.11646/zootaxa.1426.1.2

De Wit P. \& Erséus C. 2010. Genetic variation and phylogeny of Scandinavian species of Grania (Annelida: Clitellata: Enchytraeidae), with the discovery of a cryptic species. Journal of Zoological Systematics and Evolutionary Research 48: 285-293. https://doi.org/10.1111/j.1439-0469.2010.00571.x

De Wit P., Rota, E. \& Erséus C. 2009. Grania (Annelida: Clitellata: Enchytraeidae) of the Great Barrier Reef, Australia, including four new species and a re-description of Grania trichaeta Jamieson, 1977. Zootaxa 38: 16-38.

De Wit P., Rota, E. \& Erséus C. 2011. Phylogeny and character evolution in Grania (Annelida, Clitellata). Zoologica Scripta 40: 509-519. https://doi.org/10.1111/j.1463-6409.2011.00486.x

Diaz R.J., Erséus C. \& Boesch D.F. 1987. Distribution and ecology of Middle Atlantic Bight Oligochaeta. Hydrobiologia 155: 215-225. https://doi.org/10.1007/BF00025654

Eisen G. 1904. Enchytraeidae of the West Coast of North America. Harriman Alaska Expedition, Alaska XII. Doubleday, Page \& Co., New York. Available from

https://www.biodiversitylibrary.org/bibliography/11673\#/summary [accessed 13 Dec. 2017].

Erséus C. 1974. Grania pusilla sp. n. (Oligochaeta, Enchytraeidae) from the west coasts of Norway and Sweden with some taxonomic notes on the genus Grania. Sarsia 56: 87-94.

https://doi.org/10.1080/00364827.1974.10411264

Erséus C. 1976. Marine subtidal Tubificidae and Enchytraeidae (Oligochaeta) of the Bergen area, western Norway. Sarsia 62: 25-48. https://doi.org/10.1080/00364827.1976.10411312

Erséus C. 1977. Marine Oligochaeta from the Koster area, west coast of Sweden, with descriptions of two new enchytraeid species. Zoologica Scripta 6: 293-298.

https://doi.org/10.1111/j.1463-6409.1978.tb00781.x

Erséus C. 1980. A new species of Grania (Oligochaeta, Enchytraeidae) from Ascension Island, South Atlantic. Sarsia 65: 27-28. https://doi.org/10.1080/00364827.1980.10431468

Erséus C. 1990. Marine Oligochaeta of Hong Kong. In: Morton B. (ed.) Proceedings of the Second International Marine Biological Workshop: The Marine Flora and Fauna of Hong Kong and Southern China: 259-335. Hong Kong University Press, Hong Kong.

Erséus C. \& Lasserre P. 1976. Taxonomic status and geographic variation of the marine enchytraeid genus Grania Southern (Oligochaeta). Zoologica Scripta 5: 121-132.

https://doi.org/10.1111/j.1463-6409.1976.tb00689.x

Erséus C. \& Lasserre P. 1977. Redescription of Grania monochaeta (Michaelsen), a marine enchytraeid (Oligochaeta) from South Georgia (SW Atlantic). Zoologica Scripta 6: 299-300.

https://doi.org/10.1111/j.1463-6409.1978.tb00782.x

Erséus C. \& Rota E. 2003. New findings and an overview of the oligochaetous Clitellata (Annelida) of the North Atlantic deep sea. Proceedings of the Biological Society of Washington 116 (4): 892-900.

Erséus C., Rota, E., Matamoros L. \& De Wit P. 2010. Molecular phylogeny of Enchytraeidae (Annelida, Clitellata). Molecular Phylogenetics and Evolution 57 (2): 849-858.

https://doi.org/10.1016/j.ympev.2010.07.005 
Hagen G. 1954. Michaelsena achaeta nov. sp. ein neuer Oligochaet aus der Kieler Bucht. Faunistische Mitteilungen aus Norddeutschland 1: 12-13.

Healy B. 1996a. The distribution of Oligochaeta on an exposed rocky shore in southeast Ireland. Hydrobiologia 334: 51-62. https://doi.org/10.1007/BF00017353

Healy B. 1996b. New species of Marionina (Oligochaeta: Enchytraeidae) from a wave-exposed rocky shore in SE Ireland. Journal of Natural History 30: 1287-1295.

https://doi.org/10.1080/00222939600771221

Healy B. \& Coates K.A. 1999. Finding enchytraeid oligochaetes (Clitellata) in hot climates: species occurrence on the shores of Bermuda. Hydrobiologia 406: 111-117.

https://doi.org/10.1007/978-94-011-4207-6_11

Jamieson B.G.M. 1977. Marine meiobenthic Oligochaeta from Heron and Wistari Reefs (Great Barrier Reef) of the genera Clitellio, Limnodriloides and Phallodrilus (Tubificidae) and Grania (Enchytraeidae). Zoological Journal of the Linnean Society 61: 329-349.

https://doi.org/10.1111/j.1096-3642.1977.tb01032.x

Kennedy C. 1966. A taxonomic revision of the genus Grania (Oligochaeta: Enchytraeidae). Journal of Zoology 148: 399-407. https://doi.org/10.1111/j.1469-7998.1966.tb02959.x

Knöllner F.H. 1935. Ökologische und systematische Untersuchungen über litorale und marine Oligochäten der Kieler Bucht. Zoologische Jahrbücher (Systematik) 66: 425-512.

Kossmagk-Stephan K.J. 1983. Marine Oligochaeta from a sandy beach of the Island of Sylt (North Sea) with description of four new enchytraeid species. Mikrofauna des Meeresbodens 89: 593-618.

Kossmagk-Stephan K.J. 1985. Systematik, Faunistik und Lebenszyklus mariner Oligochaeta der Nordund Ostseeküste. Master's Dissertation. Georg-August-Universität, Germany.

Lasserre P. 1966. Oligochètes marins des côtes de France. I. Bassin d'Arcachon: Systématique. Cahiers de Biologie Marine 7: 295-317.

Lasserre P. 1967. Oligochètes marins des côtes de France. II. Roscoff, Penpoull, étangs saumâtres de Concarneau: systématique, écologie. Cahiers de Biologie Marine 8: 273-293.

Lasserre P. 1971. The Marine Enchytraeidae (Annelida, Oligochaeta) of the eastern coast of North America with notes on their geographical distribution and habitat. Biological Bulletin 140: 440-460.

Locke J.M. 2000. Ultrastructure of the statocyst of the marine enchytraeid Grania americana (Annelida: Clitellata). Invertebrate Biology 119: 83-93. https://doi.org/10.1111/j.1744-7410.2000.tb00176.x

Locke J.M. \& Coates K.A. 1998. A new species of Grania (Enchytraeidae, Clitellata, Annelida) and redescription of Grania pusilla from a rocky shore in SE Ireland. Journal of Natural History 32: 11011114. https://doi.org/10.1080/00222939800770551

Locke J.M. \& Coates K.A. 1999. Redescriptions of Grania americana, G. bermudensis and descriptions of two new species of Grania (Annelida: Clitellata: Enchytraeidae) from Bermuda. Proceedings of the Biological Society of Washington 112: 598-623.

Locke J.M. \& Coates K.A. 2000. An illustrated key to the species of Grania and Randidrilus (Annelida: Clitellata: Enchytraeidae) of eastern North America, Bermuda, and the Caribbean area. Proceedings of the Biological Society of Washington 113: 617-632.

Michaelsen W. 1888. Die Oligochaeten von Süd-Georgien nach Ausbeute der deutschen Station von 1882-83. Jahrbuch der Hamburgischen wissenschaftlichen Anstalt 5: 53-73.

Michaelsen W. 1900. Das Tierreich, Vol. 10: Oligochaeta. Friedländer \& Sohn, Berlin. 
Michaelsen W. 1907. Oligochaeta. Die Fauna Südwest-Australiens 1 (2):117-232.

Michaelsen W. 1921. Neue und wenig bekannte Oligochäten aus skandinavischen Sammlungen. Arkiv för Zoologi 13: 1-25.

Nielsen C.O. \& Christensen B. 1959. The Enchytraeidae - critical revision and taxonomy of European species (Studies on Enchytraeidae VII). Natura Jutlandica 8-9: 1-160.

Pierantoni U. 1901. Sopra una nuova specie di oligochete marino (Enchytraeus macrochaetus n. sp.). Monitore Zoologico Italiano 12: 201-202.

Pierantoni U. 1903. Studii anatomici su Michaelsena macrochaeta Pierant. Mitteilungen aus dem Zoologischen Station zu Neapel 16: 409-444.

Pierantoni U. 1915. Per l'identità di Grania maricola Southern con Michaelsena macrochaeta Pierant. Bollettino della Società dei Naturalisti in Napoli 27: 1-10.

Prantoni A.L., Di Domenico M. \& Lana P.C. 2014. A taxonomic overview of marine and estuarine oligochaetes from Brazil. Marine Biodiversity 44: 275-278. https://doi.org/10.1007/s12526-013-0190-3

Prantoni A.L., De Wit P. \& Erséus C. 2016. First reports of Grania (Clitellata: Enchytraeidae) from Africa and South America: molecular phylogeny and descriptions of nine new species. Zoological Journal of the Linnean Society 176: 485-510. https://doi.org/10.1111/zoj.12333

Rodriguez P. 1986. Nuevos resultados acerca de la fauna de Oligoquetos acuáticoas del País Vasco y cuenca altal del Ebro II. Enchytraeidae. Munibe Ciencias Naturales Natur Zientziak 38: 81-87.

Rota E. 1995. Italian Enchytraeidae (Oligochaeta). I. Bolletino di Zoologia 62 (2): 183-231. https://doi.org/10.1080/11250009509356067

Rota E. \& Erséus C. 1996. Six new species of Grania (Oligochaeta, Enchytraeidae) from the Ross Sea, Antarctica. Antarctic Science 2: 169-183. https://doi.org/10.1017/S0954102096000247

Rota E. \& Erséus C. 1997. A re-examination of Grania monochaeta (Michaelsen) (Oligochaeta: Enchytraeidae), with descriptions of two new species from Subantarctic South Georgia. Journal of Natural History 31: 27-42. https://doi.org/10.1080/00222939700770031

Rota E. \& Erséus C. 2000. Two new and peculiar species of Grania (Annelida: Clitellata: Enchytraeidae) inhabiting Tasmanian estuaries. New Zealand Journal of Zoology 27: 245-254.

https://doi.org/10.1080/03014223.2000.9518232

Rota E. \& Erséus C. 2003. New records of Grania (Clitellata, Enchytraeidae) in the Northeast Atlantic (from Tromsø to the Canary Islands), with descriptions of seven new species. Sarsia 88: 210-243. https://doi.org/10.1080/00364820310001615

Rota E., Erséus C. \& Wang H. 2003. Grania ocarina sp. n., G. darwinensis (Coates and Stacey) comb. n., and other marine Enchytraeidae (Oligochaeta) from the Dampier area, Western Australia. In: Walker D. \& Jones D. (eds) The Marine Flora and Fauna of Dampier, Western Australia: 497-511. Western Australian Museum, Perth.

Rota E., Wang H. \& Erséus C. 2007. The diverse Grania fauna (Clitellata: Enchytraeidae) of the Esperance area, Western Australia, with descriptions of two new species. Journal of Natural History 41: 999-1023. https://doi.org/10.1080/00222930701391682

Shurova N.M. 1979. Enchitreidy (Oligochaeta) dal'nevostocnych morej SSSR [Enchytraeids of the far eastern seas of the USSR]. Issledovannja Pelagiceskich i Donnych Organismov Dal'nevostocnych Morej, Vladivostok: 75-90. 
Southern R. 1913. Clare Island Survey, Part 48: Oligochaeta. Proceedings of the Royal Irish Academy 31: $1-15$.

Stephenson J. 1930. The Oligochaeta. Clarendon Press, Oxford.

Stephenson J. 1932. Oligochaeta. Part I. Microdrili. Discovery Reports 4: 233-264.

van Haaren T. 2016. Oligochaeten van brakke en zoute wateren in Nederland (Annelida: Oligochaeta). Nederlandse Faunistische Mededelingen 46: 155-164.

Manuscript received: 25 May 2017

Manuscript accepted: 5 July 2017

Published on: 29 December 2017

Topic editor: Rudy Jocqué

Desk editor: Danny Eibye-Jacobsen

Printed versions of all papers are also deposited in the libraries of the institutes that are members of the EJT consortium: Muséum national d'Histoire naturelle, Paris, France; Botanic Garden Meise, Belgium; Royal Museum for Central Africa, Tervuren, Belgium; Natural History Museum, London, United Kingdom; Royal Belgian Institute of Natural Sciences, Brussels, Belgium; Natural History Museum of Denmark, Copenhagen, Denmark; Naturalis Biodiversity Center, Leiden, the Netherlands; Museo Nacional de Ciencias Naturales-CSIC, Madrid, Spain; Real Jardín Botánico de Madrid CSIC, Spain. 